\title{
Living donor liver transplantation at the Pusan National University Yangsan Hospital, Korea: result of living donor hepatectomy in a single center
}

\author{
Hyo Jung Ko, Je Ho Ryu, Kwangho Yang, Byung Hyun Choi, Tae Beom Lee, Jae Ryong Sim
}

Division of Hepatobiliary, Department of Surgery, Pusan National University Yangsan Hospital, Yangsan, Korea

Background: Living donor liver transplantation is currently the most feasible treatment method for patients with end-stage liver disease. We discuss the results of living donor hepatectomy in our single center for 10 years, including donor characteristics and postoperative outcomes, and directions for improvement.

Methods: The 261 living donors underwent liver donation surgery were reviewed retrospectively from May 2010 to May 2020 at Pusan National University Yangsan Hospital.

Results: The ages of donors ranged from 16 to 64 years old, and 182 male and 79 female. Liver grafts were five types, 110 (44.9\%) in caudal middle hepatic vein trunk preserved right lobe, 105 (42.5\%) in modified right lobe, $16(6.5 \%)$ in extended right lobe, 14 $(5.7 \%)$ in left lobe, and one (0.4\%) in right posterior segment. The average weight of the graft was $686.2 \mathrm{~g}$, the volume was $37.5 \%$, and the average of body mass index of the donor was $23.4 \mathrm{~kg} / \mathrm{m}^{2}$. Of 261 donors, there was no mortality and no postoperative hepatic failure or multi-organ failure. The average operation time was 372.7 minutes, the mean length of hospital stay was 13.7 days, the intensive care unit (ICU) stay was 1.02 days, and the intraoperative transfusion rate was $1.5 \%$. Postoperative complications occurred in 44 donors. According to Clavien-Dindo classification, grade I complications occurred in 29 patients (11.1\%), grade II in one patient (0.4\%), grade III in 15 patients $(5.7 \%)$ and there was no case of grade IV and V. Grade III or more severe complications requiring invasive procedure or surgical treatment were seven cases of biliary stenosis or leakage, five portal stenosis, one duodenal ulcer bleeding, one ascites, and one bleeding.

Conclusions: Although limitations of retrospective data and short follow-up period, postoperative outcome of liver donor was relatively good. Further study with long-term follow-up and minimally invasive and refined surgical techniques to reduce minor complications are needed.

Corresponding author: Je Ho Ryu

E-mail: ryujhhim@hanmail.net

(c) The Korean Society for Transplantation

This is an Open Access article distributed under the terms of the Creative Commons Attribution Non-Commercial License (http://creativecommons.org/licenses/by-nc/4.0/) which permits unrestricted non-commercial use, distribution, and reproduction in any medium, provided the original work is properly cited. 